\title{
Semantic Orientation of Sentiment Analysis on Social Media \\ ${ }^{1}$ Sri Khetwat Saritha, ${ }^{2}$ Devshriroy \\ 1,2 Department of CSE \& IT, Maulana Azad National Institute of Technology Email: ${ }^{1}$ skmanit@gmail.com, ${ }^{2}$ droy.iit@gmail.com
}

\section{ABSTRACT}

User Generated Contents on social media, such has Blogs, Forums, YouTube, Twitter, Facebook and so on contains opinions or sentiments generated by the users about the object, such has product reviews, movie reviews, book reviews etc. The texts in these social media sites are short and generated constantly. These contents are well suited for knowledge discovery. The purpose of this paper is to locate, extract, classify and summarize the customer opinion about the products from the social media site YouTube. The proposed framework determines the semantic orientation of opinion expressed on product features as positive, negative or neutral. The system is also integrated with a visualization module to present feature based summary of user generated contents.

\section{Indexing terms/Keywords}

User Generated Contents, Social Media, Opinion Mining, Sentiment analysis, Alchemy API.

\section{Council for Innovative Research}

Peer Review Research Publishing System

Journal: International Journal of Computers \& Technology

\author{
Vol 11, No.4 \\ editor@cirworld.com \\ www.cirworld.com, member.cirworld.com
}




\section{INTRODUCTION}

A number of review sources are available in the Web where reviews about product can be found, like in $\mathrm{E}-\mathrm{Commerce}$ sites (Amazon), forums, blogs, and social media site like YouTube, Facebook, Twitter and so on. These sources are very popular among the customers. A landscape of the social media [26] is shown in the figure 1.

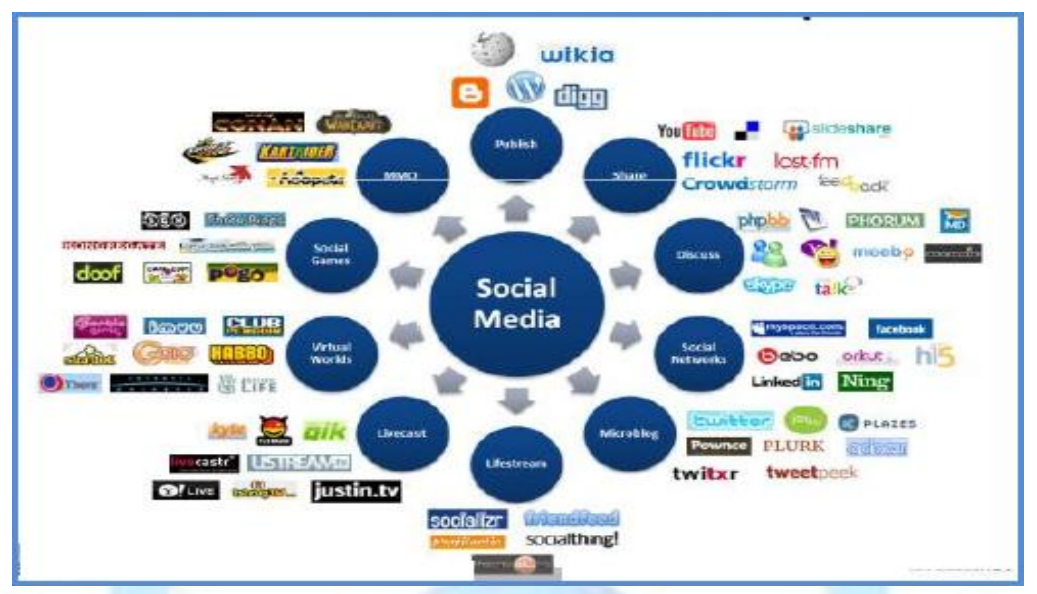

Figure 1: Social Media Landscape

Online selling and purchasing of products are increasing day by day. Customers give their views regarding the product under customer's review generally which includes customer's natural language. These reviews are known as user generated contents.

Customers generally express their views in natural language. For example "Size of Nokia Lumia is very good and handy". The feature "size and handy" of the mobile "Nokia Lumia" is highlighted. Mostly a customer expresses opinion about a product as a whole or about its features as shown in figure 2. Such customer reviews helps the company to know and analyze the public or customer opinions about their products to establish future directions for improvement [1].

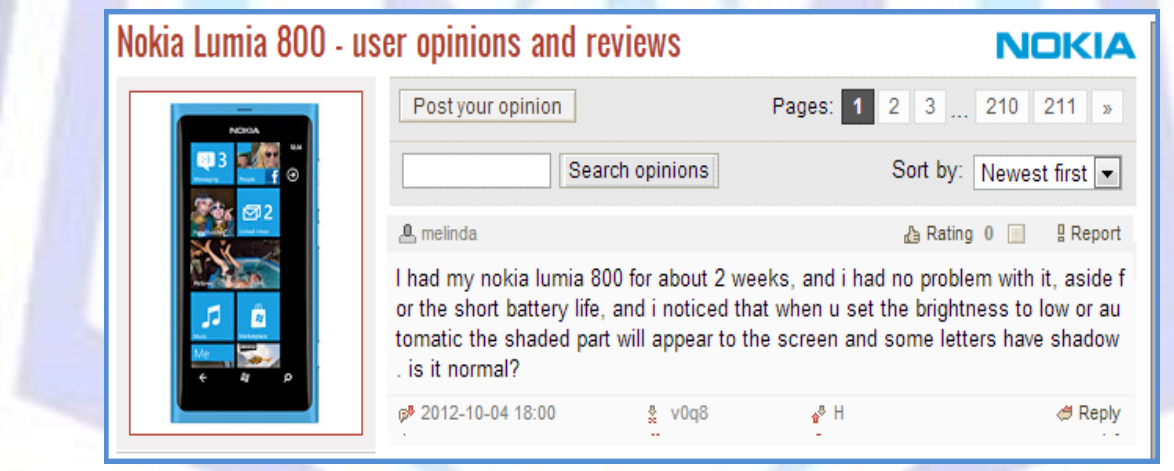

Figure 2: Review on Nokia Lumia 800 (example)

Important thing in classifying reviews is the choice of feature set. The feature based opinion extraction is a task related to information extraction, which consists in extracting structured opinions on features of some object from subjective texts [2] The problem of feature based sentiment extraction is divided into two different tasks [3]:

(1) Features of the product about which the reviewer have expressed their opinions are to be identified and extracted.

(2) The semantic orientations or the polarity of the opinions are to be determined.

The semantic orientation $[4,5]$ or polarity of a term indicates the positive or negative implications of that term being used in an opinion. In this paper, a feature based sentiment analysis framework is shown to determine opinions that are expressed on different features of the product.

\section{USER GENERATED CONTENT RESOURCES}

User generated contents holds the information about a product. It can be collected from various blogs, review sites, micro blogging and social media sites like YouTube, Twitter, Facebook and so on. This text is unstructured and unmanaged which needs proper arrangement to extract knowledge from it.

\section{(1) Review Sites}

Review site[6] is a website on which reviews can be posted about businesses, people, product or services. Early review sites included Epinions.com and Amazon.com. 


\section{(2) Blogs}

Due to continuous grow, the number of blogs doubles every 5months and there are new blogs published every second [7]. A Blog[8] is a discussion or information site published on the World Wide Web and consisting of discrete entries ("posts") typically displayed in reverse chronological order (the most recent appears first).

\section{(3) Social Media}

There are many social media sources available on World Wide Web, among them

YouTube [9] allows billions of users to discover, upload and share originally created videos. It provides forum for people to connect, inspire others across the globe.

Twitter [10] is an online social networking service and micro blogging service. This service connects to latest stories, ideas, opinions and news. It enables its users to send and read text - based messages up to 140 characters, known as "tweets".

Flickr [11] is a photo sharing websites where anyone can upload and tag photos, browse others' photos, and add comments and annotations. Users can create photo set and collections to manage content and participate in topical groups to cultivate a sense of community.

\section{OPINION MINING AND SENTIMENT ANALYSIS}

The increasing user generated contents on the internet forced researchers to study the problem of opinion mining. It is a growing research area in natural language processing, computational linguistic, text mining and information retrieval. Opinion mining is a research domain deals with the methods of detecting and extracting opinions and sentiments from the user generated contents.

The definition of opinion mining was proposed by Pang \& lee [6]. Opinion is a private state of a person thinking about something [12]. Finding opinion about the product from online sites is a very big task because finding the reaction of the customers from the large review set becomes difficult for the customer if goes manually by reading all reviews, which is time consuming. As these contents are unstructured and are unmanaged. Figure 3 shows the different opinions expressed by the customer about the product and there features.

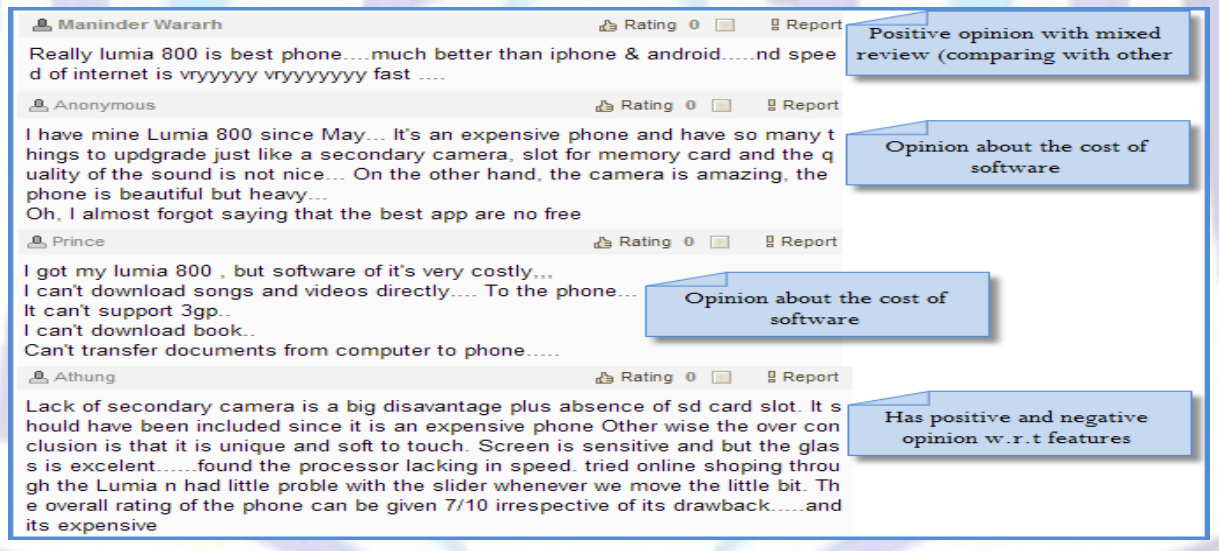

Figure 3: Semantic orientation of reviews

Opinion mining from user generated contents can be done at document level, sentence level and entity and feature or aspect level. There are two types of opinion [13] - Regular opinions which target at some entities/features and Comparative opinion which make comparison of more than one entity, as shown in figure 4.

it's a beautiful phone with a very nice os a lot more good looking than iphone or android i have also used an android phone for quite some time and the battery backup of the lumia is really excellent.... as for the need to have zune for transfering files it will be removed in the next update of windows phone 8 ....

also guys even the iphone cant send files over bluetoot but we dont see anyone complaining. it ados to security of the phone... totally value for money

Figure 4: Holding Opinion by comparing with other 
In Figure 5 the negative opinion expressed by the customer on a product is shown.

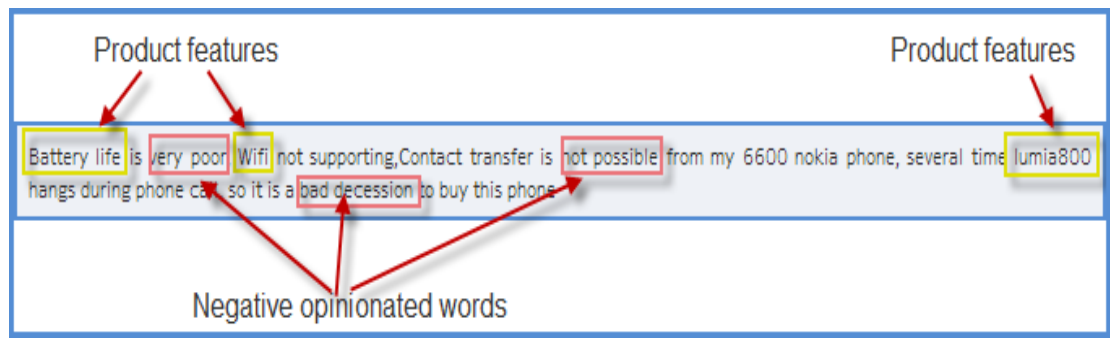

Figure 5: Customer stating Negative Opinion on few features

In some reviews the whole text does not represent opinion but only a portion of a review or some sentences include opinion oriented words as shown in figure 6.

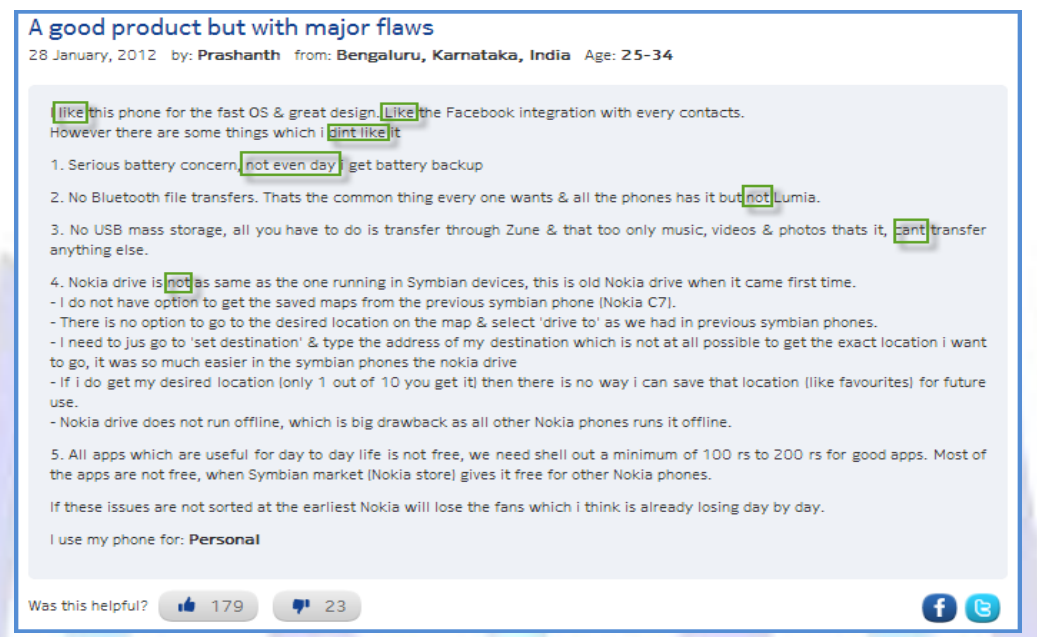

Figure 6: Lengthy review with few Semantic orientations

There are many application areas where sentiment analysis can be used, such as[14]analysis on financial markets, analysis on products, analysis on location (tourism), analysis on elections, analysis on movies and software programs, and so on.

\section{PREVIOUS WORKS}

Various mining techniques in extracting opinions generated by the user on different entities/features are studied by the researchers and various approaches are given by the researchers.

A heuristic feature based extraction algorithm given in [15] depends on feature terms with their number of occurrence. They use association rule mining based on Apriori algorithm to extract frequent item sets as explicit product features. In this paper we have used Apriori algorithm to count the occurrence of the feature terms in the user generated text. An ontology based opinion mining extraction system given in [16], this system worked semantically. They constructed the ontology manually and updated as new features were added.

An unsupervised information extraction system called OPINES was developed by [17] which mine product reviews to build a model of product features, their evaluation by reviewers and their relative quality across products. A heuristics based feature extraction algorithm is given in [18], they used association rule mining based on the Apriori algorithm to extract frequent item sets as explicit product feature. A rule based approach for feature extraction is given in [19], where they extracts large number of features from the reviews and removes multiple conflicting words in a sentence.

A semantic orientation of phrases was developed by [20] they proposed a PMI - IR algorithm to estimate the semantic orientation of a phrase. Based on POS tagging [21], the author carried out the extraction of frequent features, opinion words and infrequent features through the co-occurrence relationship among them.

In this paper, a framework is designed to determine the semantic orientation of opinion expressed on product features as positive, negative or neutral. We locate, extract, classify and summarize the customer opinion about the products from the social media site YouTube.

\section{OPINION EXTRACTION SYSTEM}

The framework involves various steps in extracting the opinions from the user generated contents. They are Crawling, Preprocessing and Semantic Orientation. 


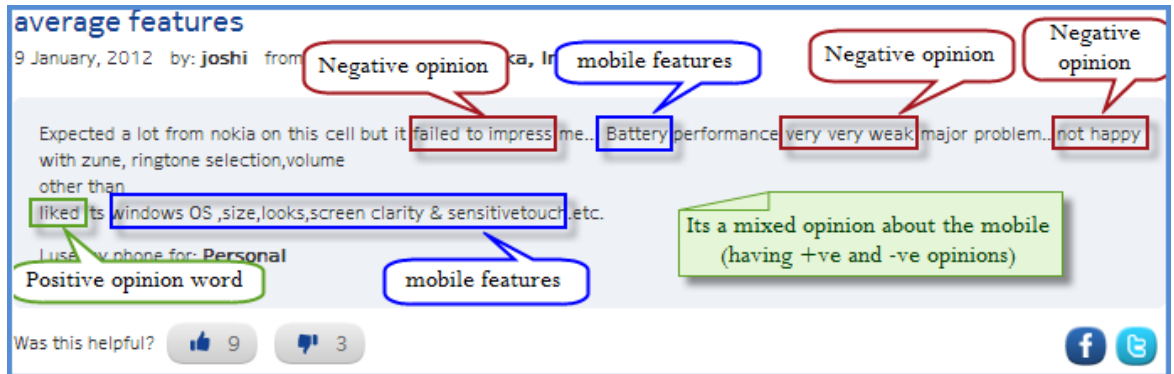

Figure7. Semantic orientation and features of mobile in a review

\section{(4) Crawling}

Web Crawler is a computer program that browses the World Wide Web in a methodical, automated manner or in an orderly fashion [22]. It is relatively simple automated program, or script, that methodically scans or crawls through Internet pages to create an index of the data [23]. Extraction of UGC done by crawling YouTube based on the request of the user is shown in the figure 7.

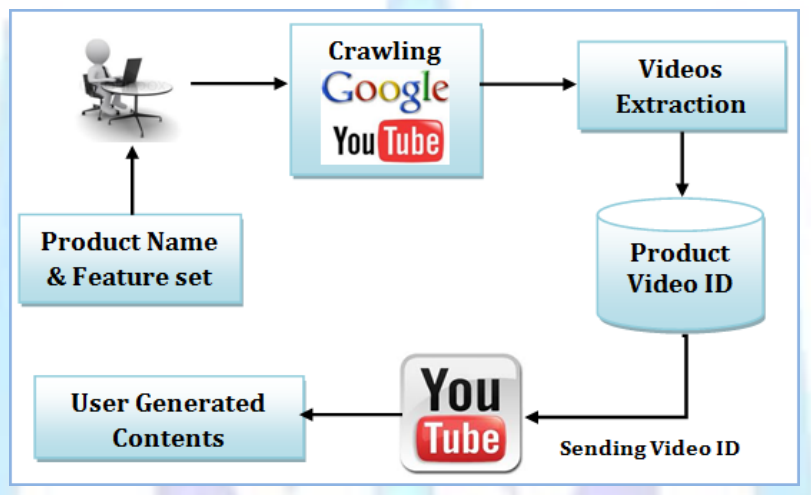

Figure 8: Extraction of UGC from YouTube

The Videos are searched by sending a query to the YouTube server using product name as the filter. All Video ID are downloaded which have their unique Id number.

"http://gdat.youtube.com/feeds/api/videos" videos"

For Example:

\begin{tabular}{|l|l|l|}
\hline Produce Name & Feature Set & Video ID \\
\hline Nokia Lumia & Battery Life & 20XFhQpmh2E \\
& & msbE0LxpYKs \\
& & QvnDunLJSPE \\
& & MJyqURfRJ4w \\
& & YS9azQLWNAI \\
& & and so on. \\
\hline
\end{tabular}

For each unique video id, a request is sent which returns the XML file with comments.

"http://gdata.youtube.com/feeds/api/videos/VIDEOID/comments". 
The comments are extracted within the <comment> tag. An Algorithm 1 shown below describes the extraction of reviews from YouTube:

\section{Algorithm 1: Extract Reviews from YouTube.}

Step 1: Input \{product name, feature set\}

Step 2: Searching for the video from YouTube by sending a query to the YouTube Server URL:

http://gdata.youtube.com/feeds/api/videos

Step3: Extract all Video Ids based on the product name as a Filter.

Output: $\{$ VideolD.txt $\}$

Step 4: Store all features into a file. \{Feature.txt\}

Step 5: For each VideoID in VideolD.txt perform step 6 to step 8

Step 6: Read the VidoID and send each Video ID to URL

http://gdata.youtube.com/feeds/api/video/VIDEOID/comments

Step 7: Return XML file with comments

Step 8: Read the XML and extract the text within <comment> tag

Output: $\{$ comment.txt\}

\section{Preprocessing and Semantic Orientation}

While processing the text, stop words are removed and stemming is done. The unwanted symbols are removed using ASCII check for each word. Figure 9 shows the Extraction of semantic concepts of the User Generated Contents.

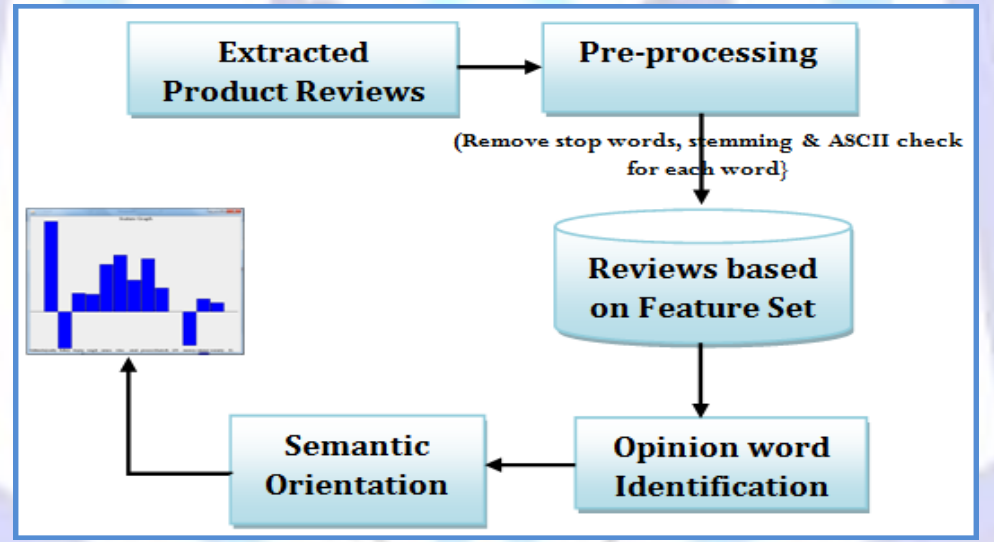

Figure 9: Extraction of Filtered UGC based on feature set from YouTube and Semantic Orientation of the comments

After the removing the noise from the reviews, the reviews are separated based on the feature set given by the user. For opinion word identification and semantic orientation AlchemyAPI is used [24]. AlchemyAPI provides easy-to-use mechanisms to identify positive / negative sentiment within any document or web page. The "URLGetTextSentiment" call is utilized to extract positive / negative sentiments. The result obtained in XML form.

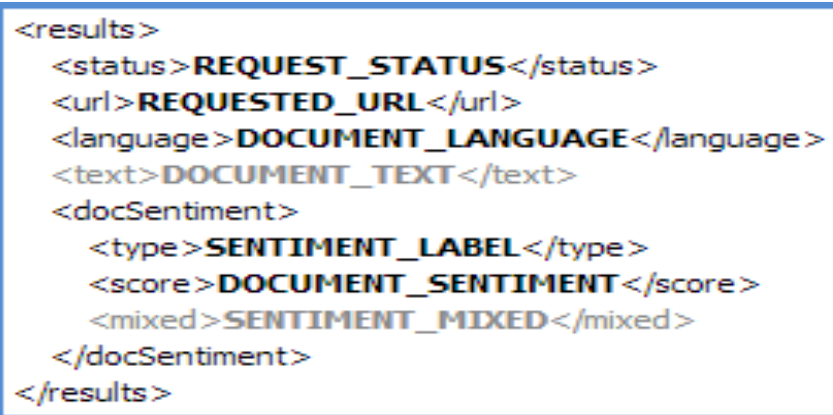

Figure 10: Response format of AlchemyAPI call (XML) 
The algorithm shown below shows the extraction system and semantic orientation of the User generated contents.

\section{Algorithm 2: Preprocessing of Extract Reviews from YouTube.}

Step 1: Process the comment file to remove unwanted symbols using the ASCll check for each word.

Step 2: Identify the keywords of feature file in the comment file.

Input: $\{$ comment.txt, feature.txt\}

Step3: Classify the comments in comment.txt and create a separate file for each feature say battery.txt, camera.txt and so on for the product: mobile phone

Step 4: For each comment file generated in Step 3 perform Step 5 to Step 6

Step 5: Perform the stemming of words in each feature file containing comments, identify the Opinion words

Input: \{battery. Txt, camera.txt, ... $\}$

Step 6: Calculating the Semantic Orientation of the comments, built using Alchemy API.

Step 7: Create a Bar graph with feature on $\mathrm{X}$-axis and the polarity score on $\mathrm{Y}$-axis.

\section{RESULTS AND DISCUSSION}

Mobiles phones relatively have a small set of features. We annotated the reviews crawled from YouTube based on the request of the user. We extracted around 40 features which are in common from the reputed mobile selling sites. An interface was designed where the user can input the product name and the features set for which he would like to know the opinion of the other customers about the product on given feature set.

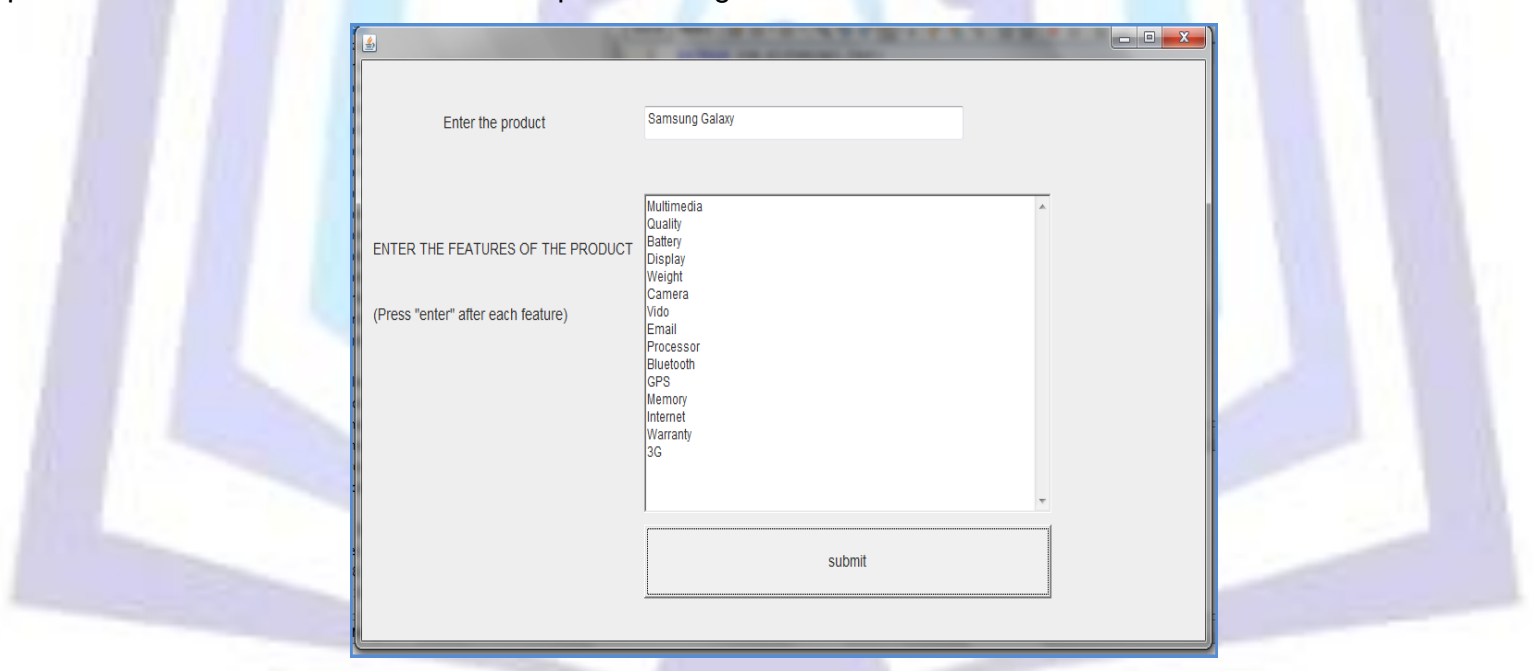

Figure 11: Interface for entering the Product name and required featured set

AlchemyAPI[25] utilizes natural language processing technology and machine learning algorithms to analyze content, extracting semantic meta-data: information about people, places, companies, topics, facts \& relationships, authors, languages and more. API endpoints are provided for performing content analysis on Internet-accessible web pages, posted HTML or text content. 


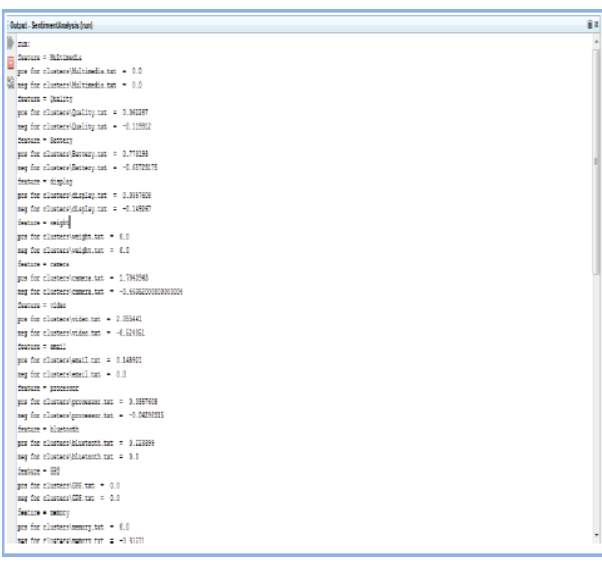

(a)

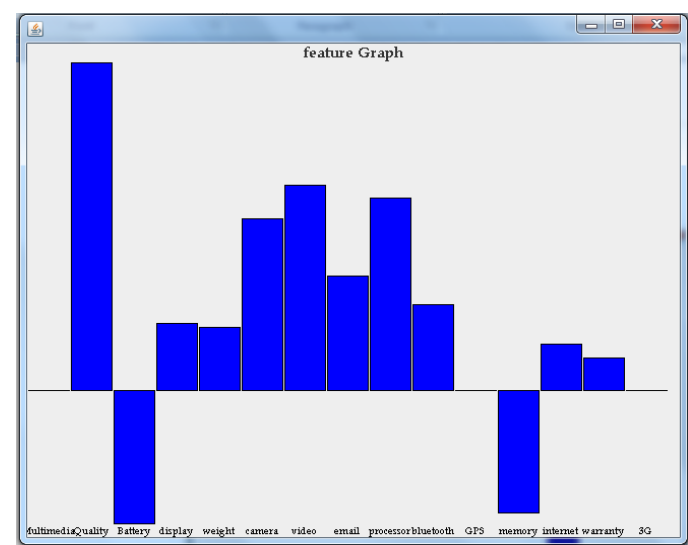

(b)

Figure 12: (a) Feature set and their score (semantic orientation), (b) Visualization of the opinion extraction system based on features

We analyzed around 30 mobile phones and their features. We received a good result for the given product and feature set. Figure 6 shows the result for one for the product - Samsung Galaxy.

\section{CONCLUSION}

This paper describes the method for extracting the comments from the YouTube based on the user request. The main idea behind this work was to determine the semantic orientation of customer opinion about the products so that one can easily know about the pros and cons of a product and can make decision accordingly.

\section{REFERENCES}

[1] N.Li and Z.Ping, "Consumer Online Shopping Attitudes and Behaviour: An assessment of Research", Proceedings $8^{\text {th }}$ Americas Conference Inf. Syst., 2002, pp. $508-517$.

[2] Fermín L.Cruz, José A. Troyano, Fernando Enríquez, F.Javier Ortega, Carlos G.Vallejo,

"A Knowledge Rich Approach to Feature - Based Opinion Extraction from Product Reviews", SMUC'10, October 30, 2010, Toronto, Ontario, Cananda. pp. $13-20$.

[3] B.Liu, "Opinion Mining", Web Data Mining: Exploring Hyperlinks, Contents, and Usage Data, Springer, 2007, pp 411 448

[4] Lehrer, Adrienne, "Semantic fields and lexical structure/A.Lehrer. North - Holland; American Elsevier, Amsterdam: New York:, 1974.

[5] Fermín L. Cruz, José A. Troyano, Fernando Enríquez, F. Javier Ortega, Carlos G. Vallijo, "A knowledge Rich Approach to Feature - bas opinion extraction from product reviews", SMUC'10, Oct 30, 2010, Toronto, Ontario, China.

[6] www.en.wikipedia. Ord/wiki/Review_site

[7] Antonio S. Valderrábanos, Enrique Torrejón,"Natural Opinions: NLP based opinion extraction in user generated content", CEUR Workshop Proceedings (CEUR-WS.org), Vol 697/ pp 16 - 20.

[8] www.en.wikipedia.org/wiki/Blogs

[9] www.youtube.com/t/about_youtube

[10] en.wikipedia.org/wiki/Twitter

[11] http://net.educause.edu/ir/library/pdf/ELI7034.pdf

[12] Bo Pang and Lillian Lee, "opinion mining and sentiment analysis", Foundation and trends in Information retrieval Vol 2, Nos. 1 - 2 (2008) 1 - 135 2008, 2008

[13] http://www.cs.uic.edu/ liub/FBS/Sentiment-Analysis-tutorial-AAAl-2011.pdf

[14] Ion Smeureanu, Cristian Bucur, "Applying supervised opinion mining techniques on online user reviews", Informatical Economica vol. 16. No. 2/2012 pp. 81 - 91

[15] Hu, M., Liu, B.: Mining and Summarizing Customer Reviews, In Proc. 10th Int. Conf. Knowledge Discovery and Data Mining, Seattle, WA, 2004, 168-177 
[16] Silvana Aciar, Debbie Zhang, Simeon Simoff, and John Debenham "Informed Recommender: Basing Recommendations on Consumer Product Reviews", IEEE Intell. Syst., Vol 22, no. 3, 2007, pp. 39-47

[17] Popescu, A. M., Etzioni, O.: Extracting Product Features and Opinions from Reviews, In Proc. Conf. Human Language Technology and Empirical Methods in Natural

[18] Language Processing, Vancouver, British Columbia, 2005, 339-346.Hu, M., Liu, B.: Mining and Summarizing Customer Reviews, In Proc. 10th Int. Conf. Knowledge Discovery and Data Mining, Seattle, WA, 2004, 168-177

[19] X.Ding, B.Liu and T.Philip, "A Holistic Lexicon - Based Approah to Opinion Mining", Proc. Int. conf. Web Search Web Data Mining, 2008, pp, $231-240$

[20] Peter D. Turney, "Thums up or Thumbs Down? Semantic Orientation Applied to Unsupervised classification of Reviews", Proceedings of the $40^{\text {th }}$ Annual meeting of the Association for Computational Linguistics (ACL), Philadelphia, July 2002, pp. $417-424$

[21] M.Q. Hu and B. Liu, "Mining opinion features in customer reviews", in proceedings of $19^{\text {th }}$ National Conference on Artificial Intelligence (AAAI - 2004), San Jose, USA, 2004

[22] en.wikipedia.ord/wiki/Web_crawler

[23] www.wisegreek.com/what-is-a-web-crawler.htm

[24] http://www.alchemyapi.com/api/sentiment/

[25] http://www.alchemyapi.com/api/ 